\title{
Research Article Spectral Classification of Non-Coaxiality for Two-Dimensional Incremental Stress-Strain Response
}

\author{
Jiangu Qian, ${ }^{1,2,3}$ Jean-Pierre Bardet $^{3}$ and Maosong Huang ${ }^{1,2}$ \\ ${ }^{1}$ Department of Geotechnical Engineering, Tongji University, Shanghai 200092, China \\ ${ }^{2}$ Key Laboratory of Geotechnical and Underground Engineering of Ministry of Education, \\ Tongji University, Shanghai 200092, China \\ ${ }^{3}$ Sonny Astani Department of Civil and Environmental Engineering, University of Southern California, \\ Los Angeles, CA 90089-2531, USA
}

Correspondence should be addressed to Jiangu Qian, qianjiangu@tongji.edu.cn

Received 31 August 2010; Accepted 30 December 2010

Academic Editor: K. R. Rajagopal

Copyright (C) 2010 Jiangu Qian et al. This is an open access article distributed under the Creative Commons Attribution License, which permits unrestricted use, distribution, and reproduction in any medium, provided the original work is properly cited.

The present study examines the non-coaxial aspects of incremental material behavior, and attempts to classify the incremental non-coaxiality that relates stress and strain increments. In the solid mechanics literature, non-coaxiality (NC) refers usually to incremental strains and stress states having different principal directions. Departing from conventional non-coaxiality, the analysis investigates the incremental non-coaxiality (INC) of linearized rate-type solids. This study uses the concept of deviatoric second-order work for examining the relations between stability and incremental non-coaxiality. Based on a spectral analysis of the constitutive compliance matrix, it proposes three classifications for distinguishing various degrees of incremental non-coaxiality and stability. These classifications determine the conditions for the existence of incremental coaxiality (i.e., colinearity of stress and strain increments), stability, instability, and stable-instable transition (i.e., positive, negative, or zero second-order deviatoric work). The study illustrates these classifications in the cases of generic elastic and elastoplastic constitutive models. The analysis pertains to two-dimensional cases. Additional research is required to extend the analysis from two to three dimensions.

\section{Introduction}

The anisotropic and non-coaxial behaviors of geomaterials are challenging to model using the conventional flow theory of plasticity, which assumes that the strain increments and principal stress have identical direction, that is, are coaxial [1-3]. For instance, the associative flow rule of plasticity, which assumes that strain increments are normal to the yield surface, disagrees with many experimental evidences $[4,5]$ and micromechanical observations $[6,7]$, 


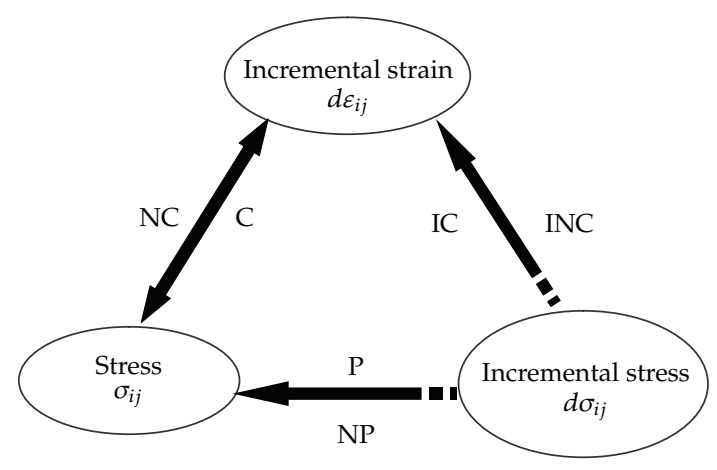

\footnotetext{
INC: Incremental non-coaxial

IC: Incremental coaxial

P: Proportional loading

NP: Non-proportional loading

NC: Non-coaxial

C: Coaxial
}

Figure 1: Illustration of relationship among incremental strain, incremental stress and stress.

which show non-coaxiality, that is, different principal directions for stress states and strain increments.

As early as Hill [8], several theories have been proposed to introduce noncoaxiality. For instance, one approach added tangent plasticity to classical coaxial models $[1,9]$. Another approach defined strain increment in terms of stress states and material anisotropy, which may lead to an anisotropic hardening law [10-12]. Others have described noncoaxial behaviors using double-shear models [13-15]. Micromechanical studies have related noncoaxiality to the anisotropic fabric resulting from the arrangement of material particles and associated voids $[16,17]$.

Most studies of non-coaxiality focused on the case of non-proportional loading, and assumed coaxiality under proportional loading [18]. However, experiments showed that anisotropic solids, such as geomaterials, may exhibit considerable degrees of noncoaxiality even under proportional loading $[5,19,20]$. This results in two distinct mechanisms relevant to non-coaxiality. According to theoretical [21,22] and experimental studies [5, 20], noncoaxiality is largely induced by tangent yield effects under non-proportional loading. For proportional loading, experiments showed that non-coaxiality tends to result from induced or inherent material anisotropy $[5,19]$. To distinguish the two different mechanisms, this analysis expands the definition of conventional non-coaxiality to incremental non-coaxiality, which is hereafter referred to as the difference between principal directions of strain and stress increments.

Figure 1 illustrates the relationship among non-coaxiality, incremental non-coaxiality and stress path. It relates these three concepts using (1) stress state $\sigma_{i j}$, (2) stress increment $d \sigma_{i j}$, and (3) strain increment $d \varepsilon_{i j}$. The principal direction angles of $\sigma_{i j}, \varepsilon_{i j}$, and $d \varepsilon_{i j}$ are defined as $\theta_{\sigma}, \theta_{d \sigma}$ and $\theta_{d \varepsilon}$, respectively (Figure 2). Like in the solid mechanics literature [23], Figure 1 defines coaxiality $\left(\mathrm{C}, \theta_{\sigma}=\theta_{d \varepsilon}\right)$ and non-coaxiality $\left(\mathrm{NC}, \theta_{\sigma} \neq \theta_{d \varepsilon}\right)$ in terms of $\sigma_{i j}$ and $d \varepsilon_{i j}$. Proportional (P, $\theta_{\sigma}=\theta_{d \sigma}$ ) loading corresponds to $d \sigma_{i j}$ proportional to $\sigma_{i j}$, and 


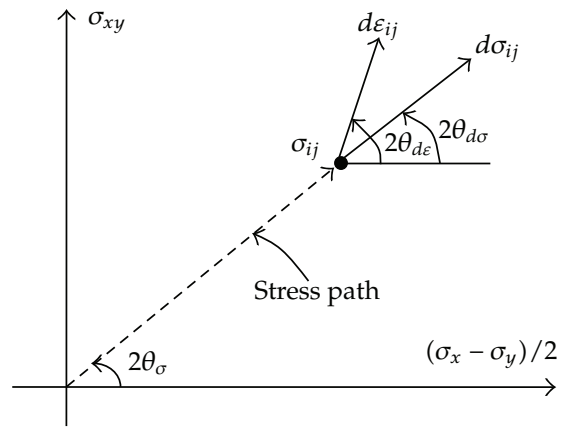

(a) Incremental non-coaxiality with proportional loading $\left(\theta_{d \varepsilon} \neq \theta_{d \sigma}=\theta_{\sigma}\right)$

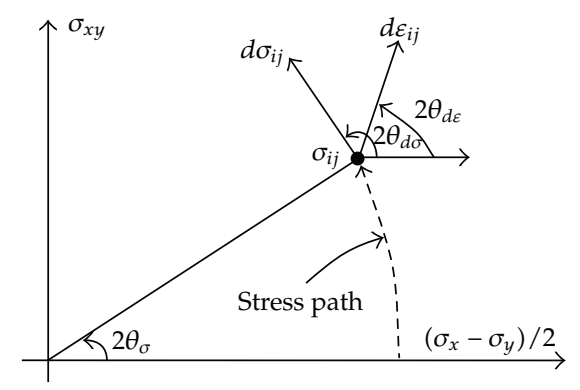

(b) Incremental non-coaxiality with non-proportional loading $\left(\theta_{d \varepsilon} \neq \theta_{d \sigma} \neq \theta_{\sigma}\right)$

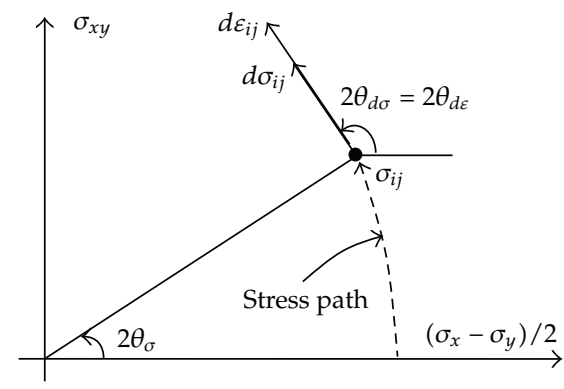

(c) Incremental coaxiality with non-proportional loading $\left(\theta_{d \varepsilon}=\theta_{d \sigma} \neq \theta_{\sigma}\right)$

Figure 2: Non-coaxiality available by three approaches: (a) INC+P; (b) INC+NP; (c) IC+NP.

non-proportional (NP, $\left.\theta_{\sigma} \neq \theta_{d \sigma}\right)$ loading refers to changes in principal direction of $d \sigma_{i j}$, when $\sigma_{i j}$ moves along nonlinear stress paths. Incremental coaxiality (IC, $\left.\theta_{d \sigma}=\theta_{d \varepsilon}\right)$ and incremental non-coaxiality (INC, $\theta_{d \sigma} \neq \theta_{d \varepsilon}$ ) are defined in terms of $d \sigma_{i j}$ and $d \varepsilon_{i j}$. Therefore, NC can be generated in three different ways through INC + P (Figure 2(a)), INC + NP (Figure 2(b)) and IC + NP (Figure 2(c)) .

The incremental non-coaxiality (INC) and non-proportionality (NP) of loading can be defined, respectively, as

$$
\begin{gathered}
\theta_{\text {inc }}=\theta_{d \varepsilon}-\theta_{d \sigma}, \\
\theta_{\text {np }}=\theta_{d \sigma}-\theta_{\sigma} .
\end{gathered}
$$

Therefore, non-coaxiality (NC), incremental non-coaxiality (INC), and non-proportionality (NP) of loading are related by

$$
\theta_{\mathrm{nc}}=\theta_{\mathrm{inc}}+\theta_{\mathrm{np}}
$$

where $\theta_{\text {np }}$ can be determined from the prescribed loading path and $\theta_{\text {inc }}$ is a type-rate material property that needs to be measured from incremental stress-strain response. Clearly, $\theta_{\mathrm{nc}}=\theta_{\mathrm{inc}}$ in the particular case of proportional loading (Figure 2(a)). 


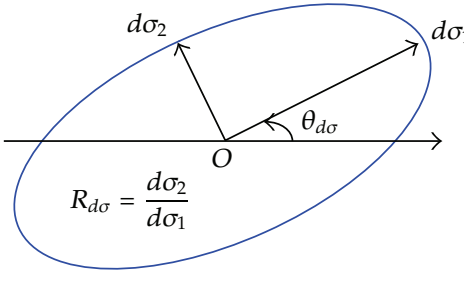

(a) Incremental stress ellipse

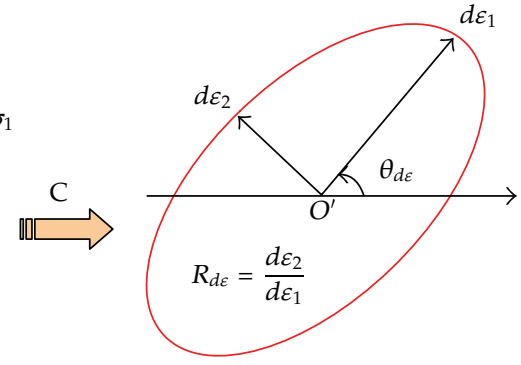

(b) Incremental strain ellipse

Figure 3: Lame's visualization of incremental stress-strain responses.

For stress-controlled problems, the above considerations restrict our attention to incremental non-coaxiality (INC). The present analysis focuses on two aspects: (1) for which materials incremental non-coaxiality (INC) may become negligible or large and (2) in which direction materials may preserve incremental coaxiality (IC). The analysis is presented in two dimensions, but can be extended to three-dimensions with some additional effort.

\section{Lame's Representation of Incremental Non-Coaxiality}

Material behaviors are modeled using rate-type linearized solids, that is,

$$
d \varepsilon_{i j}=C_{i j k l} d \sigma_{k l}
$$

where $d \varepsilon_{i j}$ is the incremental strain, $d \sigma_{i j}$ is the incremental stress, and $C_{i j k l}$ is the incremental compliance tensor that represents some underlying anisotropy state. The indices are $i, j \ldots=$ 1,2 in two-dimensions. $C_{i j k l}$ can be written in matrix form as follows:

$$
[C]=\left[\begin{array}{lll}
C_{1111} & C_{1122} & C_{1112} \\
C_{2211} & C_{2222} & C_{2212} \\
C_{1211} & C_{1222} & C_{1212}
\end{array}\right] .
$$

The physical meaning of incremental non-coaxiality can be illustrated using Lame's ellipse (Figure 3), which represents a two-dimensional second-order tensor using an inclined ellipse. $d \sigma_{i j}$ is uniquely expressed in terms of principal stress values $d \sigma_{1}$ and $d \sigma_{2}\left(d \sigma_{1} \geq d \sigma_{2}\right)$ and the orientation angle $\theta_{d \sigma}$ of principal direction. Similarly, the strain increment $d \varepsilon_{i j}$ is defined using the principal values of strain increment $d \varepsilon_{1}$ and $d \varepsilon_{2}\left(d \varepsilon_{1} \geq d \varepsilon_{2}\right)$ and $\theta_{d \varepsilon}$ the orientation angle of principal direction. The incremental response is coaxial when $\theta_{d \varepsilon}=\theta_{d \sigma}$ and non-coaxial when $\theta_{d \varepsilon} \neq \theta_{d \sigma}$. 
Table 1: Orientation angle and ratio of principal stress increments.

\begin{tabular}{lcc}
\hline Stress increments matrix & $\theta_{d \sigma}$ & $R_{d \sigma}$ \\
\hline$\left[\begin{array}{cc}d \sigma_{1} & 0 \\
0 & d \sigma_{2}\end{array}\right]$ & 0 & $\frac{d \sigma_{1}}{d \sigma_{2}}$ \\
{$\left[\begin{array}{cc}d \sigma_{2} & 0 \\
0 & d \sigma_{1}\end{array}\right]$} & $\pi / 2$ & $\frac{d \sigma_{2}}{d \sigma_{1}}$ \\
{$\left[\begin{array}{cc}-d \sigma_{1} & 0 \\
0 & d \sigma_{2}\end{array}\right]$} & $\pi / 2$ & $-\frac{d \sigma_{1}}{d \sigma_{2}}$ \\
{$\left[\begin{array}{cc}d \sigma_{1} & 0 \\
0 & -d \sigma_{2}\end{array}\right]$} & 0 & $-\frac{d \sigma_{1}}{d \sigma_{2}}$ \\
{$\left[\begin{array}{cc}-d \sigma_{1} & 0 \\
0 & -d \sigma_{2}\end{array}\right]$} & & $\frac{d \sigma_{1}}{d \sigma_{2}}$ \\
\hline
\end{tabular}

In Lame's representation, (2.1) is restated as follows:

$$
\begin{aligned}
\theta_{d \varepsilon} & =f\left(d \sigma_{1}, d \sigma_{2}, \theta_{d \sigma}\right), \\
d \varepsilon_{1} & =f_{2}\left(d \sigma_{1}, d \sigma_{2}, \theta_{d \sigma}\right), \\
d \varepsilon_{2} & =f_{3}\left(d \sigma_{1}, d \sigma_{2}, \theta_{d \sigma}\right),
\end{aligned}
$$

where the nonlinear functions $f_{1}, f_{2}$, and $f_{3}$ depend on $C_{i j k l}$ and $-90^{\circ} \leq \theta_{d \sigma} \leq 90^{\circ}$.

Without loss of generality, (2.3) may be simplified assuming $d \sigma_{2}=1$ and $d \varepsilon_{2}=1$ (see the appendix)

$$
\begin{gathered}
\theta_{\mathrm{inc}}=\theta_{d \varepsilon}-\theta_{d \sigma}=F_{1}\left(R_{d \sigma}, \theta_{d \sigma}\right), \\
R_{d \varepsilon}=\frac{d \varepsilon_{1}}{d \varepsilon_{2}}=F_{2}\left(R_{d \sigma}, \theta_{d \sigma}\right),
\end{gathered}
$$

where functions $F_{1}$ and $F_{2}$ are analogous to $f_{1}, f_{2}$ and $f_{3}$, and $R_{d \sigma}=d \sigma_{1} / d \sigma_{2}$ denotes the ratio of incremental principal stresses.

Mapping uniquely a stress increment onto a Lame's ellipse requires assigning a positive sign to principal values. Otherwise, different stress increments would correspond to the same Lame's ellipses. For instance, assuming $d \sigma_{1}>d \sigma_{2}>0$, Table 1 shows five tensors that have the same Lame's ellipse but different values of $\theta_{d \sigma}$ and $R_{d \sigma}$.

\section{Classifications of Incremental Non-Coaxiality}

Three different classifications are proposed for incremental non-coaxiality, based on the values of $C_{i j k l}$ coefficients that indicate some kind of fabric anisotropy. These classifications addresses the following two issues: (1) relation of stability and INC, and (2) number of directions that preserve incremental coaxiality, that is, stress and strain increments have identical or opposite directions. 


\subsection{Classification 1: Energy-Based Classification for INC}

The first classification intends to distinguish between materials with strong or weak INC, noting that the degree of INC is intrinsically related to material stability. Since the strain increment has the same principal direction as its deviatoric part, the deviatoric constitutive equation is considered

$$
d e_{i j}=C_{i j k l}^{d} d \sigma_{k l}
$$

where $d e_{i j}=d \varepsilon_{i j}-\delta_{i j} d \varepsilon_{k k} / 2$, and $C_{i j k l}^{d}=C_{i j k l}-\delta_{i j} C_{a a k l} / 2$.

The eigenvalues of $C_{i j k l}^{d}$ can be analytically calculated by solving a quadratic equation, which is simpler than solving a cubic equation for the eigenvalues of $C_{i j k l}$. This is because $C_{i j k l}^{d}$ is rank deficient and always possess one zero eigenvalue, leaving only two eigenvalues to determine.

The deviatoric second-order work is

$$
d^{2} W^{d}=d \sigma_{i j} d e_{i j}=d s_{i j} d e_{i j}=\left|d s_{i j}\right|\left|d e_{i j}\right| \cos 2\left(\theta_{d \varepsilon}-\theta_{d \sigma}\right)=\left|d s_{i j}\right|\left|d e_{i j}\right| \cos 2 \theta_{\text {inc }},
$$

where $d s_{i j}$ is the deviatoric part of $d \sigma_{i j},\left|d s_{i j}\right|$ and $\left|d e_{i j}\right|$ denote the magnitude of $d s_{i j}$ and $d e_{i j}$, respectively. One obtains:

$$
\cos 2 \theta_{\mathrm{inc}}=\frac{d s_{i j} d e_{i j}}{\left|d s_{i j}\right|\left|d e_{i j}\right|}=\frac{d \sigma_{i j} C_{i j k l}^{d} d \sigma_{k l}}{\left|d s_{i j}\right|\left|C_{i j k l}^{d} d \sigma_{k l}\right|} .
$$

The trivial cases $d s_{i j}=0$ and $d e_{i j}=0$ can be ignored without loss of generality. In general, there are three independent deviation angles between the principal axes of stress and strain increments in three-dimensional case, for which $\theta_{\text {inc }}$ given by (3.3) does not represent a certain angular orientation of incremental non-coaxiality. However, $\cos \theta_{\text {inc }}$ is an energybased parameter that can be extended to higher-dimensional problems.

Assuming $d \sigma_{2}=1, d \sigma_{i j}$ can be explicitly expressed as

$$
[d \sigma]=\frac{d \sigma_{a a}}{2}[\delta]+[d s]=\frac{R_{d \sigma}+1}{2}\left[\begin{array}{ll}
1 & 0 \\
0 & 1
\end{array}\right]+\frac{R_{d \sigma}-1}{2}\left[\begin{array}{cc}
\cos 2 \theta_{d \sigma} & \sin 2 \theta_{d \sigma} \\
\sin 2 \theta_{d \sigma} & -\cos 2 \theta_{d \sigma}
\end{array}\right]
$$

which implies that $d s_{i j}$ and $d \sigma_{i j}$ have the same principal directions.

In the particular case $C_{i j k l}^{d} \delta_{k l}=0$, that is,

$$
C_{1211}+C_{1222}=0, \quad C_{1111}-C_{2211}-C_{2222}+C_{1122}=0,
$$


which corresponds to pressure increments producing no deviatoric strains. With $C_{i j k l}^{d} \delta_{k l}=0$, equation (3.3) becomes

$$
\cos 2 \theta_{\mathrm{inc}}=\frac{d s_{i j} C_{i j k l}^{d} d s_{k l}}{\left|d s_{i j}\right|\left|C_{i j k l}^{d} d s_{k l}\right|}=\frac{n_{i j} C_{i j k l}^{d} d n_{k l}}{\left|n_{i j}\right|\left|C_{i j k l}^{d} n_{k l}\right|},
$$

where $n_{i j}$ is

$$
[n]=\left[\begin{array}{cc}
\cos 2 \theta_{d \sigma} & \sin 2 \theta_{d \sigma} \\
\sin 2 \theta_{d \sigma} & -\cos 2 \theta_{d \sigma}
\end{array}\right] .
$$

According to (3.6a)-(3.6b), $\cos 2 \theta_{\text {inc }}$ is independent from $R_{d \sigma}$, and $d^{2} W^{d}\left(=d \sigma_{i j} C_{i j k l}^{d} d \sigma_{k l}\right)$ and $\cos 2 \theta_{\text {inc }}$ have identical sign.

Equation (3.1) can be equivalently expressed in matrix form as follows:

$$
[d e]=\left[C^{d}\right][d \sigma]
$$

or

$$
\left[\begin{array}{c}
\frac{d \varepsilon_{11}-d \varepsilon_{22}}{2} \\
\frac{d \varepsilon_{22}-d \varepsilon_{11}}{2} \\
\sqrt{2} d \dot{\varepsilon}_{12}
\end{array}\right]=\left[\begin{array}{ccc}
\frac{C_{1111}-C_{2211}}{2} & \frac{C_{1122}-C_{2222}}{2} & \frac{\sqrt{2}\left(C_{1112}-C_{2212}\right)}{2} \\
\frac{C_{2211}-C_{1111}}{2} & \frac{C_{2222}-C_{1122}}{2} & \frac{\sqrt{2}\left(C_{2212}-C_{1112}\right)}{2} \\
\sqrt{2} C_{1211} & \sqrt{2} C_{1222} & 2 C_{1212}
\end{array}\right]\left[\begin{array}{c}
d \sigma_{11} \\
d \sigma_{22} \\
\sqrt{2} d \sigma_{12}
\end{array}\right] .
$$

Complicated spectral analyses for nonsymmetric matrices are avoided by noting that any nonsymmetric matrix $\mathbf{A}$ satisfies $\mathbf{x}^{\mathrm{T}} \mathbf{A} \mathbf{x}=\mathbf{x}^{\mathbf{T}}\left(\left(\mathbf{A}+\mathbf{A}^{\mathrm{T}}\right) / 2\right) \mathbf{x}$ for any vector $\mathbf{x}$. Equation (3.3) can be rewritten

$$
\cos 2 \theta_{\mathrm{inc}}=\frac{d \sigma_{i j} C_{i j k l}^{d} d \sigma_{k l}}{\left|d s_{i j}\right|\left|C_{i j k l}^{d} d \sigma_{k l}\right|}=\frac{d \sigma_{i j} \bar{C}_{i j k l}^{d} d \sigma_{k l}}{\left|d s_{i j}\right|\left|C_{i j k l}^{d} d \sigma_{k l}\right|}=\frac{[d \sigma]^{T}\left[\bar{C}^{d}\right][d \sigma]}{\sqrt{[d s]^{T}[d s]} \sqrt{[d s]^{T}\left[C^{d}\right]^{T}\left[C^{d}\right][d s]}}
$$


where $\left[\bar{C}^{d}\right]=\left(\left[C^{d}\right]+\left[C^{d}\right]^{T}\right) / 2$ (i.e., $\left.\bar{C}_{i j k l}^{d}=\left(C_{i j k l}^{d}+C_{k l i j}^{d}\right) / 2\right)$ is the symmetric part of $\left[C_{d}\right]$, whose equivalent matrix form is

$$
\begin{aligned}
& {\left[\bar{C}^{d}\right]} \\
& =\left[\begin{array}{ccc}
\frac{C_{1111}-C_{2211}}{2} & \frac{C_{2211}-C_{1111}+C_{1122}-C_{2222}}{4} & \frac{\sqrt{2}\left(2 C_{1211}+C_{1112}-C_{2212}\right)}{4} \\
\frac{C_{2211}-C_{1111}+C_{1122}-C_{2222}}{4} & \frac{C_{2222}-C_{1122}}{2} & \frac{\sqrt{2}\left(2 C_{1222}+C_{2212}-C_{1112}\right)}{4} \\
\frac{\sqrt{2}\left(2 C_{1211}+C_{1112}-C_{2212}\right)}{4} & \frac{\sqrt{2}\left(2 C_{1222}+C_{2212}-C_{1112}\right)}{4} & 2 C_{1212}
\end{array}\right] .
\end{aligned}
$$

The eigenvalues of $\left[\bar{C}^{d}\right]$ are denoted by $\bar{\lambda}_{1}, \bar{\lambda}_{2}$ and $\bar{\lambda}_{3}$, and the corresponding orthogonal eigentensors are denoted by $\bar{\alpha}_{i j}, \bar{\beta}_{i j}$ and $\bar{X}_{i j}$. Any stress increments can be expressed as

$$
d \sigma_{i j}=\bar{a} \bar{\alpha}_{i j}+\bar{b} \bar{\beta}_{i j}+\bar{c} \bar{X}_{i j}
$$

where $\bar{a}, \bar{b}$, and $\bar{c}$ are the components in the eigendirections.

Equation (3.8) becomes

$$
\cos 2 \theta_{\mathrm{inc}}=\frac{\bar{a}^{2} \bar{\lambda}_{1}+\bar{b}^{2} \bar{\lambda}_{2}+\bar{c}^{2} \bar{\lambda}_{3}}{\sqrt{[d s]^{T}[d s]} \sqrt{[d s]^{T}\left[C^{d}\right]^{T}\left[C^{d}\right][d s]}}
$$

which shows that $\cos 2 \theta_{\text {inc }}$ depends on the signs of $\bar{\lambda}_{1}, \bar{\lambda}_{2}$ and $\bar{\lambda}_{3}$.

When $C_{i j k l}^{d} \delta_{k l}=0$, the eigenvalues of $\left[\bar{C}^{d}\right]$ are

$$
\bar{\lambda}_{1}=0, \quad \bar{\lambda}_{2}=\frac{A^{\prime}+\sqrt{B^{\prime}}}{2}, \quad \bar{\lambda}_{3}=\frac{A^{\prime}-\sqrt{B^{\prime}}}{2},
$$

where

$$
\begin{aligned}
& A^{\prime}=C_{1111}-C_{2211}+2 C_{1212}, \\
& B^{\prime}=\left(C_{1111}-C_{2211}-2 C_{1212}\right)^{2}+\left(2 C_{1211}+C_{1112}-C_{2212}\right)^{2} .
\end{aligned}
$$

Parameters $A^{\prime}$ and $B^{\prime}$ solely control the values of eigenvalues. Invoking that $\bar{\lambda}_{2} \geq \bar{\lambda}_{3}$, Figure 4 shows four distinct domains: 


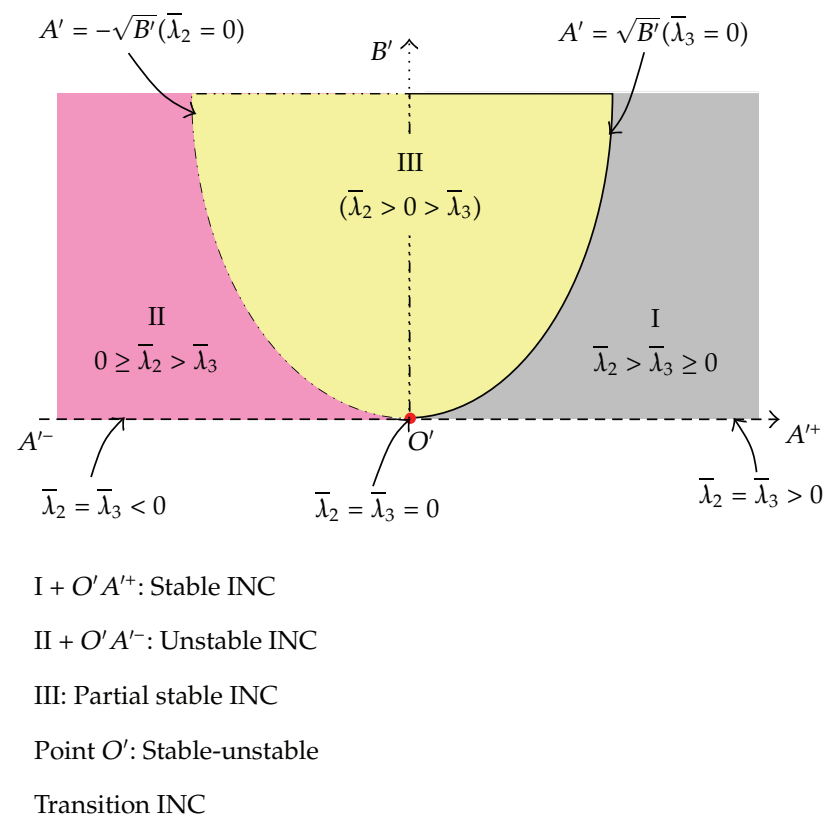

Figure 4: Classification $1\left(C_{i j k l}^{d} \delta_{k l}=0\right)$.

(I) stable; $\cos 2 \theta_{\text {inc }}>0 ; \bar{\lambda}_{1}=0$ and $\bar{\lambda}_{2} \geq \bar{\lambda}_{3} \geq 0 ; A^{\prime} \geq \sqrt{B^{\prime}}$,

(II) unstable; $\cos 2 \theta_{\text {inc }}<0 ; \bar{\lambda}_{1}=0$ and $0 \geq \bar{\lambda}_{2} \geq \bar{\lambda}_{3} ; A^{\prime}<-\sqrt{B^{\prime}}$,

(III) partially stable; $-1 \leq \cos 2 \theta_{\text {inc }} \leq 1 ; \bar{\lambda}_{1}=0$ and $\bar{\lambda}_{2}>0>\bar{\lambda}_{3} ;-\sqrt{B^{\prime}}<A^{\prime}<\sqrt{B^{\prime}}$.

Point $O^{\prime}$ : Stable-Unstable Transition; $\cos 2 \theta_{\text {inc }}=0 ; \bar{\lambda}_{1}=0$ and $\bar{\lambda}_{2}=\bar{\lambda}_{3}=0 ; A^{\prime}=B^{\prime}=0$. When $C_{i j k l}^{d} \delta_{k l} \neq 0$, the above spectral approach does not fully apply however. This case is similar to partially stable INC (Domain III in Figure 4).

\subsection{Classification 2-Spectral Classification for IC}

The second classification relates to the material directions that preserve stable incremental coaxiality (SIC) or produce unstable incremental coaxiality (UIC). The analysis looks for the principal directions (if any) of anisotropic materials, which may also represent the optimal orientation of anisotropic solids, see, for example, [24].

$C_{i j k l}^{d}$ are assumed to have three eigenvalues $\lambda_{1}, \lambda_{2}$, and $\lambda_{3}$ and three corresponding eigendirections $\alpha_{i j}, \beta_{i j}$, and $\chi_{i j}$. The eigenvalues of $C_{i j k l}^{d}$ are

$$
\lambda_{1} \equiv 0, \quad \lambda_{2}=\frac{A+\sqrt{B}}{2}, \quad \lambda_{3}=\frac{A-\sqrt{B}}{2},
$$




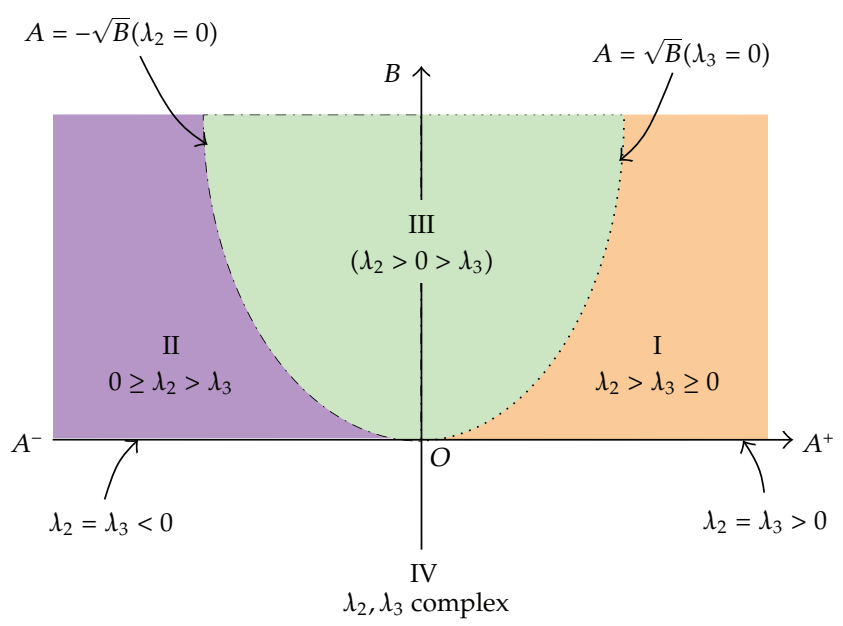
I: 4 SIC
$O A^{+}: 2$ SIC or IC
II: 4 UIC
$O A^{-}: 2$ UIC or UIC
III: 2 SIC + 2 UIC
IV: No SIC/UIC

Figure 5: Classification $2\left(C_{i j k l}^{d} \delta_{k l}=0\right)$.

where

$$
\begin{gathered}
A=\frac{C_{1111}-C_{2211}+C_{2222}-C_{1122}+4 C_{1212}}{2} \\
B=\left(\frac{C_{1111}-C_{2211}+C_{2222}-C_{1122}-4 C_{1212}}{2}\right)^{2}+4\left(C_{1112}-C_{2212}\right)\left(C_{1211}-C_{1222}\right) .
\end{gathered}
$$

Consider that a given stress increment

$$
d \sigma_{i j}=a \alpha_{i j}+b \beta_{i j}+c X_{i j}
$$

where the coefficients $a, b$, and $c$ are variables comparable to $\bar{a}, \bar{b}$ and $\bar{c}$ in (3.10). In the case $C_{i j k l}^{d} \delta_{k l}=0$ (i.e., $\alpha_{i j}=\delta_{i j}$ )

$$
d s_{i j}=b \beta_{i j}+c \chi_{i j}
$$

Thus,

$$
d e_{i j}=C_{i j k l}^{d} d \sigma_{k l}=C_{i j k l}^{d}\left(d s_{k l}+\frac{1}{2} d \sigma_{m m} \delta_{k l}\right)=C_{i j k l}^{d} d s_{k l}=b \lambda_{2} \beta_{k l}+c \lambda_{3} \chi_{k l} .
$$

Equation (3.16) implies that $d e_{i j}$ are coaxial with $d \sigma_{i j}$ when $d s_{i j}=b \beta_{i j}$ or $d s_{i j}=c X_{i j}$.

As shown in (3.13), (3.16) and Figure 5, parameters $A$ and $B$ control the values of eigenvalues $\lambda_{2}$ and $\lambda_{3}$, which determine the stability and degree of IC. The stability of IC 


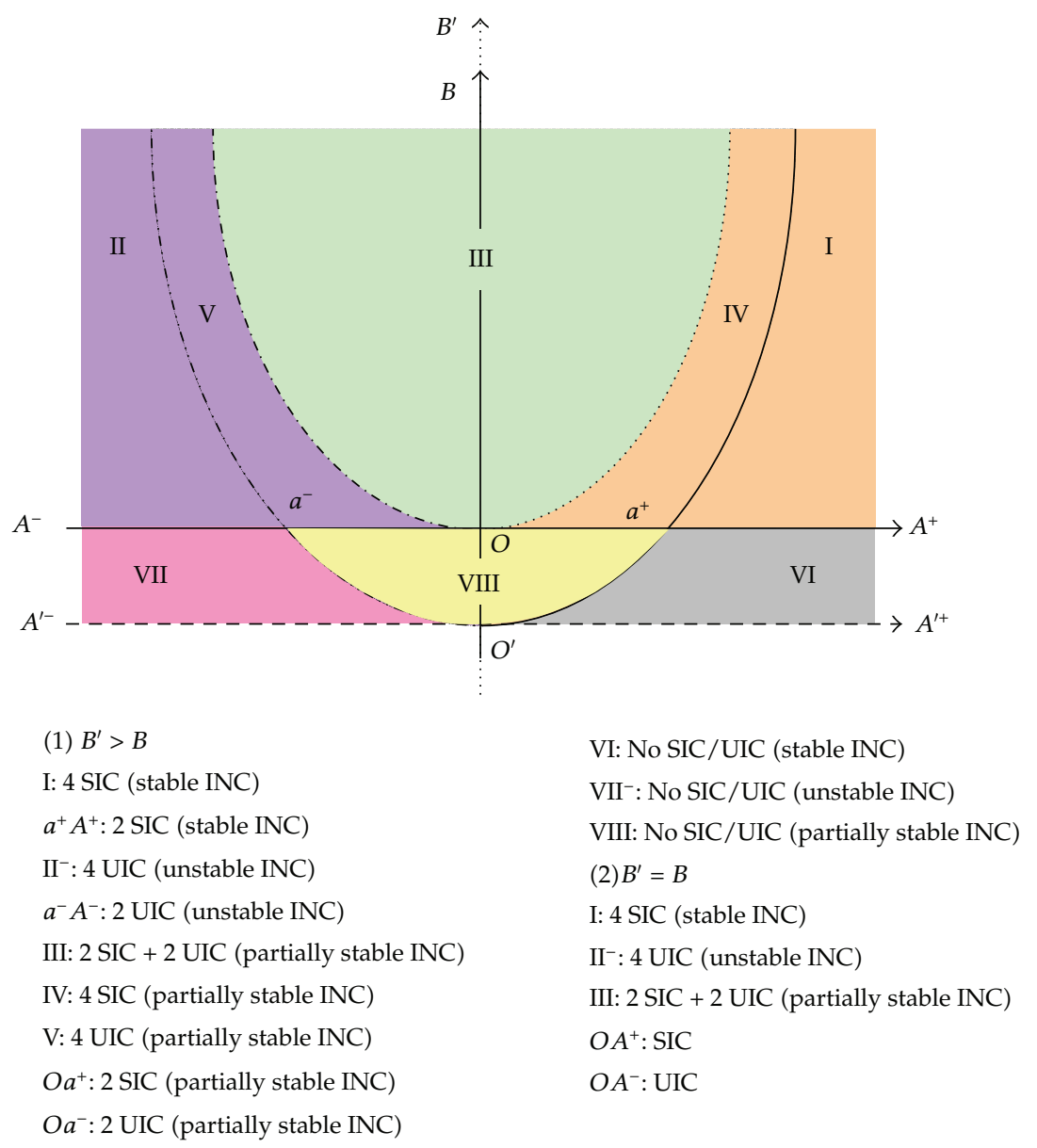

Figure 6: Classification $3\left(C_{i j k l}^{d} \delta_{k l}=0\right)$.

(i.e., SIC or UIC) depends on the signs of $\lambda_{2}$ and $\lambda_{3}$. The degree of SIC (or UIC) is indicated with the number $n$ of directions along which the incremental stress and deviator strain have identical (or opposite) direction.

\subsection{Classification 3-Couped Spectral Classification of INC}

The third classification builds upon the two previous classifications. Hereafter, we focus on the case $C_{i j k l}^{d} \delta_{k l}=0$ because $C_{i j k l}^{d} \delta_{k l} \neq 0$ creates incremental non-coaxiality (INC) in all directions without possible stable or unstable incremental coaxiality. $C_{i j k l}^{d} \delta_{k l}=0$ implies that

$$
\begin{gathered}
A^{\prime}=A, \\
B^{\prime}-B=\left(2 C_{1211}-C_{1112}+C_{2212}\right)^{2} \geq 0 .
\end{gathered}
$$

Figures 4 and 5 were combined into Figure 6 to produce the third classification, which gives information on stability as well as the number of directions for stable or unstable incremental coaxiality. Table 2 is a tabular representation of classifications in Figure 6 . This classification will be illustrated using examples in each classification domains. 


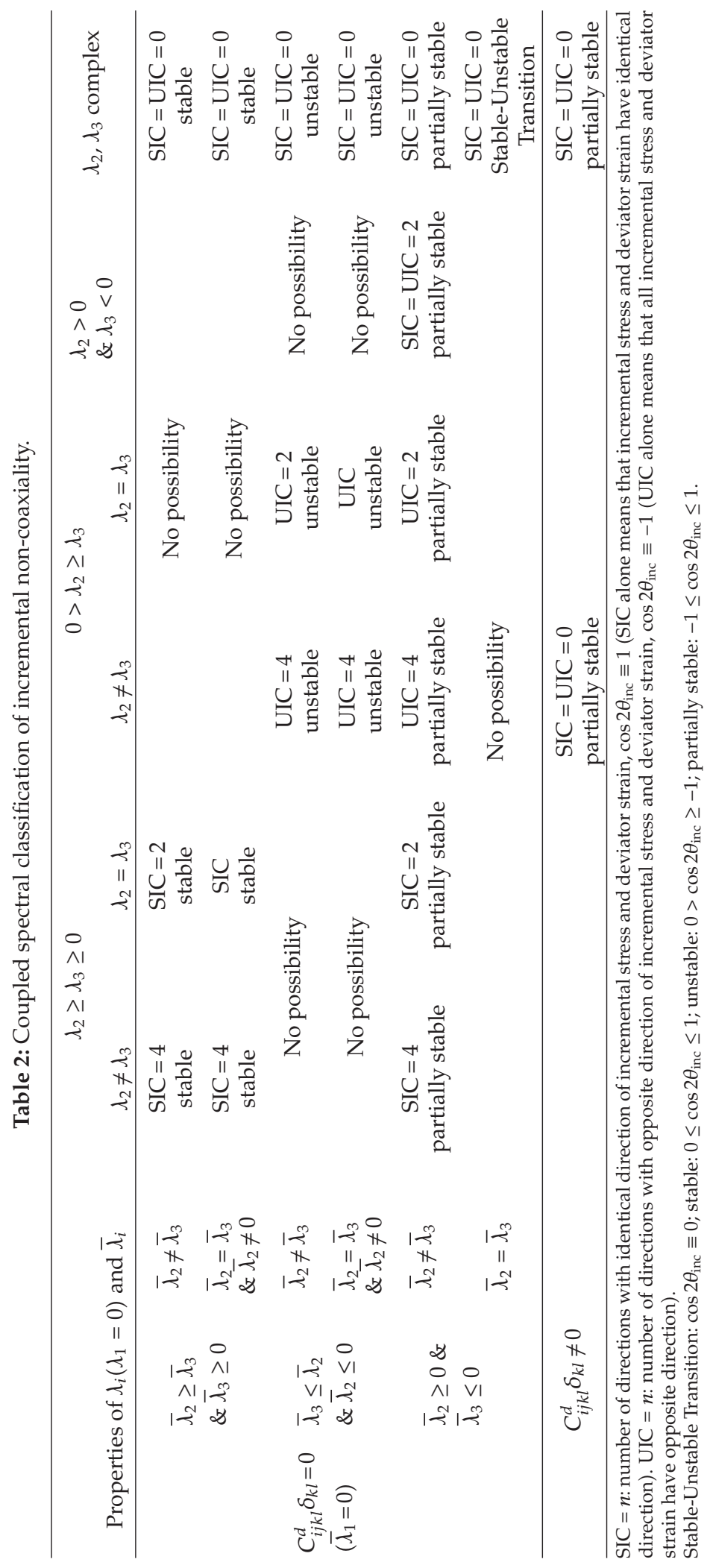




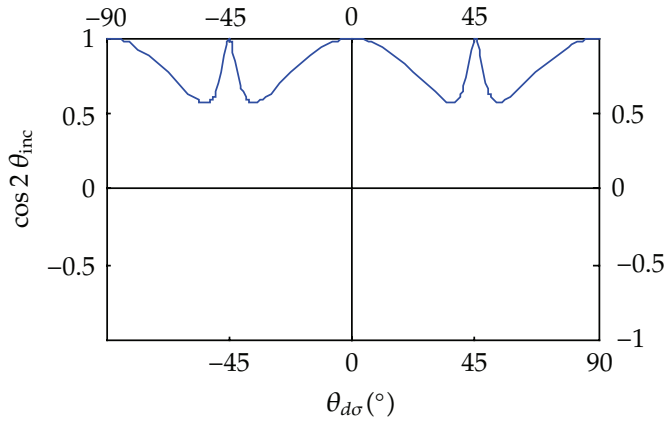

(a) 4 SIC

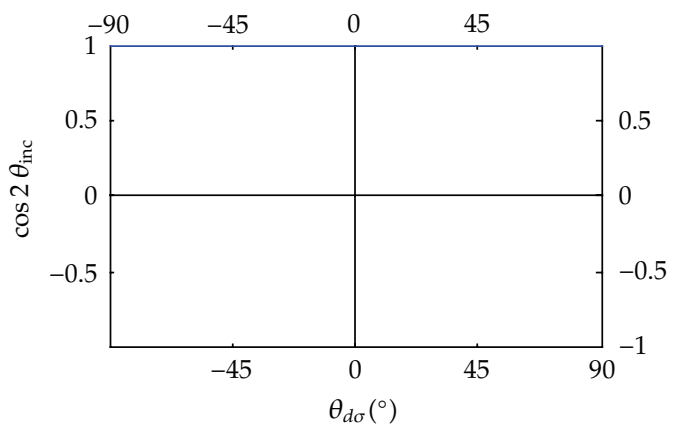

(c) SIC

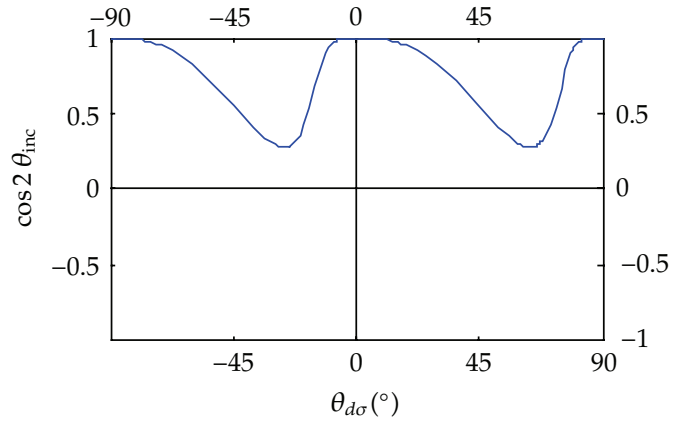

(b) 2 SIC

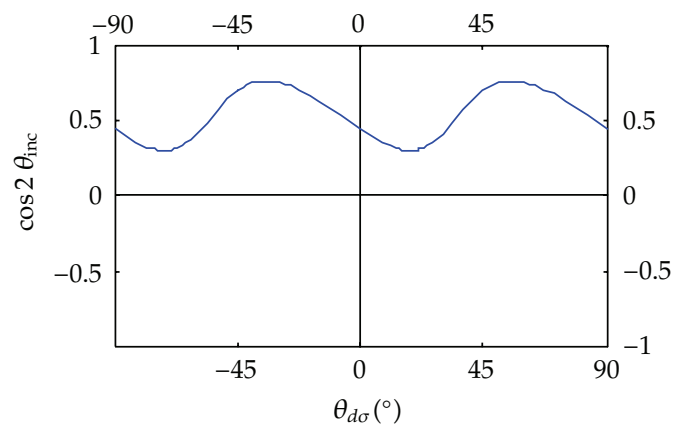

(d) No SIC

Figure 7: $\theta_{d \sigma}-\cos 2 \theta_{\text {inc }}$ for stable INC $\left(C_{i j k l}^{d} \delta_{k l}=0\right)$.

When $B^{\prime}=B$, which results in $C_{i j k l}^{d}=\bar{C}_{i j k l}^{d}$ (i.e., $\lambda_{2}=\bar{\lambda}_{2}$ and $\lambda_{2}=\bar{\lambda}_{3}$ ), points $O, O^{\prime}, a^{-}$ and $a^{+}$coincide, and Domains I, II and III remain only in Figure 6.

\section{Examples}

Incremental non-coaxiality is modeled for compliance matrices (2.2) that do not necessarily have a major symmetry, that is, $C_{i j k l} \neq C_{k l i j}$. As shown in Table 3, all components of $C_{i j k l}$ are dimensionless. With respect to distinct eigenvalue cases, examples are given to demonstrate how the classifications distinguish various degrees of incremental non-coaxiality and stability. Tables 3(a)-3(e) and Figures 7-11 contain 17 examples that apply to anisotropic elasticity as well as elastoplasticity.

Figure 7 and Table $3(\mathrm{a})$ show four cases of stable incremental non-coaxiality when $\bar{\lambda}_{2} \geq \bar{\lambda}_{3} \geq 0$. Figures 7 (a) and 7 (b) show that there are four and two stable IC (SIC) (i.e., $\cos 2 \theta_{\text {inc }}=1$ ). Figure $7($ a) falls within Domain I (Figure 6) while Figure $7(b)$ is on Boundary $a^{+} A^{+}$(Figure 6). Figure 7(c) shows a particular case of stable incremental coaxiality in all directions, which is available only if $B^{\prime}=B$, corresponding to Boundary $O A^{+}\left(\lambda_{2}=\lambda_{3}=\bar{\lambda}_{2}=\right.$ $\bar{\lambda}_{3} \geq 0$ ) in Figure 6. Finally, Figure 7(d) corresponds to complex $\lambda_{2}$ and $\lambda_{3}$ and $\bar{\lambda}_{2} \geq \bar{\lambda}_{3} \geq 0$, which falls within Domain VI (Figure 6). In this particular case, INC is stable in all directions. As illustrated in Figure 6, $\lambda_{2}>0>\lambda_{3}$ and $0>\lambda_{2} \geq \lambda_{3}$ are two cases that cannot coexist with $\bar{\lambda}_{2} \geq \bar{\lambda}_{3} \geq 0$. 
Table 3

(a) Model parameters for stable INC $\left(C_{i j k l}^{d} \delta_{k l}=0, \bar{\lambda}_{1}=\lambda_{1}=0\right)$

\begin{tabular}{lclc}
\hline $\begin{array}{l}\text { Classification } \\
\text { Domains in Figure 6 }\end{array}$ & $C_{i j k l}$ coefficients & Spectral properties & $\begin{array}{c}\text { Computation results } \\
\text { in Figure 7 }\end{array}$ \\
\hline $\mathrm{I}$ & {$\left[\begin{array}{ccc}1.5 & -0.5 & 0 \\
-0.5 & 1.5 & 0 \\
0 & 0 & 0.1\end{array}\right]$} & $\bar{\lambda}_{2}=2.0, \bar{\lambda}_{3}=0.2$, & (a) \\
$\left(B^{\prime}=B\right)$ & $\lambda_{2}=2.0, \lambda_{3}=0.2$ & \\
\hline$a^{+} A^{+}$ & {$\left[\begin{array}{ccc}1.0 & -0.5 & 1.5 \\
-1.0 & 1.5 & -1.5 \\
0 & 0 & 1.0\end{array}\right]$} & $\bar{\lambda}_{2}=3.5, \bar{\lambda}_{3}=0.5$, & (b) \\
$\left(B^{\prime}>B\right)$ & $\lambda_{2}=\lambda_{3}=2.0$ & (c) \\
\hline$O A^{+}$ & {$\left[\begin{array}{ccc}1.5 & -0.5 & 0 \\
-0.5 & 1.5 & 0 \\
0 & 0 & 1.0\end{array}\right]$} & $\bar{\lambda}_{2}=\bar{\lambda}_{3}=\lambda_{2}=\lambda_{3}=2.0$ & \\
$\left(B^{\prime}=B\right)$ & {$\left[\begin{array}{ccc}1.0 & -0.5 & 1.0 \\
-1.0 & 1.5 & -1.0 \\
-2.0 & 2.0 & 1.0\end{array}\right]$} & $\bar{\lambda}_{2}=3.0, \bar{\lambda}_{3}=1.0$ & (d) \\
\hline $\mathrm{VI}$ & & $\lambda_{2}=2.0+2.8 i($ complex) & \\
$\left(B^{\prime}>B\right)$ & & $\lambda_{3}=2.0-2.8 i$ (complex) & \\
\hline
\end{tabular}

(b) Model parameters for instable INC $\left(C_{i j k l}^{d} \delta_{k l}=0, \bar{\lambda}_{1}=\lambda_{1}=0\right)$

\begin{tabular}{|c|c|c|c|}
\hline $\begin{array}{l}\text { Classification } \\
\text { Domains in Figure } 6\end{array}$ & $C_{i j k l}$ coefficients & Spectral properties & $\begin{array}{c}\text { Computation results in } \\
\text { Figure } 8\end{array}$ \\
\hline $\begin{array}{l}\text { II } \\
\left(B^{\prime}=B\right)\end{array}$ & {$\left[\begin{array}{ccc}0.5 & 1.5 & 0 \\
1.5 & 0.5 & 0 \\
0 & 0 & -0.1\end{array}\right]$} & $\begin{array}{l}\bar{\lambda}_{2}=-0.2, \bar{\lambda}_{3}=-1.0 \\
\lambda_{2}=-0.2, \lambda_{3}=-1.0\end{array}$ & (a) \\
\hline $\begin{array}{l}a^{-} A^{-} \\
\left(B^{\prime}>B\right)\end{array}$ & {$\left[\begin{array}{ccc}0.5 & 2.0 & 0 \\
1.5 & 1.0 & 0 \\
0.5 & -0.5 & -0.5\end{array}\right]$} & $\begin{array}{l}\bar{\lambda}_{2}=-0.5, \bar{\lambda}_{3}=-1.5, \\
\lambda_{2}=\lambda_{3}=-1.0\end{array}$ & (b) \\
\hline $\begin{array}{l}O A^{-} \\
\left(B^{\prime}=B\right)\end{array}$ & {$\left[\begin{array}{ccc}0.5 & 1.5 & 0 \\
1.5 & 0.5 & 0 \\
0 & 0 & -0.5\end{array}\right]$} & $\begin{array}{l}\bar{\lambda}_{2}=\bar{\lambda}_{3}=\lambda_{2}= \\
\lambda_{3}=-1.0\end{array}$ & (c) \\
\hline VII & {$\left[\begin{array}{ccc}0.5 & 2.0 & 1.0 \\
1.5 & 1.0 & -1.0 \\
-1.5 & 1.5 & -0.5\end{array}\right]$} & $\begin{array}{l}\bar{\lambda}_{2}=-0.5, \bar{\lambda}_{3}=-1.5 \\
\lambda_{2}=-1.0+2.45 i \text { (complex) }\end{array}$ & (d) \\
\hline$\left(B^{\prime}>B\right)$ & & $\lambda_{3}=-1.0-2.45 i$ (complex) & \\
\hline
\end{tabular}

(c) Model parameters for stable-instable INC transition $\left(C_{i j k l}^{d} \delta_{k l}=0, \bar{\lambda}_{1}=\lambda_{1}=0\right)$

\begin{tabular}{|c|c|c|c|}
\hline $\begin{array}{l}\text { Classification } \\
\text { Domains in Figure } 6\end{array}$ & $C_{i j k l}$ coefficient & Spectral properties & $\begin{array}{c}\text { Computation results } \\
\text { in Figure } 9\end{array}$ \\
\hline Point $o^{\prime}$ & {$\left[\begin{array}{ccc}1.0 & 1.5 & -0.1 \\
1.0 & 1.5 & 0.1\end{array}\right]$} & $\bar{\lambda}_{2}=\bar{\lambda}_{3}=0$ & \\
\hline$\left(B^{\prime}>B\right)$ & {$\left[\begin{array}{lll}0.1 & -0.1 & 0.0\end{array}\right]$} & $\begin{array}{c}\lambda_{2}=0.2 i(\text { complex }) \\
\lambda_{3}=-0.2 i(\text { complex })\end{array}$ & \\
\hline
\end{tabular}


(d) Model parameters for partially stable INC $\left(C_{i j k l}^{d} \delta_{k l}=0, \bar{\lambda}_{1}=\lambda_{1}=0\right)$

\begin{tabular}{|c|c|c|c|}
\hline $\begin{array}{l}\text { Classification } \\
\text { Domains in Figure } 6\end{array}$ & $C_{i j k l}$ coefficients & Spectral properties & $\begin{array}{c}\text { Computation results in } \\
\text { Figure } 10\end{array}$ \\
\hline IV & {$\left[\begin{array}{ccc}1.0 & -0.5 & 0.6 \\
-1.0 & 1.5 & -0.6\end{array}\right]$} & $\bar{\lambda}_{2}=4.1, \bar{\lambda}_{3}=-0.1$ & (a) \\
\hline$\left(B^{\prime}>B\right)$ & {$\left[\begin{array}{lll}1.5 & -1.5 & 1.0\end{array}\right]$} & $\lambda_{2}=3.9, \lambda_{3}=0.1$ & \\
\hline $\begin{array}{l}o a^{+} \\
\left(B^{\prime}>B\right)\end{array}$ & {$\left[\begin{array}{rrr}1.0 & -0.5 & -4.0 \\
-1.0 & 1.5 & 4.0 \\
0.0 & 0.0 & 1.0\end{array}\right]$} & $\begin{array}{c}\bar{\lambda}_{2}=6.0, \bar{\lambda}_{3}=-2.0 \\
\lambda_{2}=\lambda_{3}=2.0\end{array}$ & (b) \\
\hline $\begin{array}{l}\mathrm{V} \\
\left(B^{\prime}>B\right)\end{array}$ & {$\left[\begin{array}{ccc}0.5 & 2.0 & 0.25 \\
1.5 & 1.0 & -0.25 \\
1.0 & -1.0 & -0.6\end{array}\right]$} & $\begin{array}{c}\bar{\lambda}_{2}=0.15, \bar{\lambda}_{3}=-2.35 \\
\lambda_{2}=-0.095, \quad \lambda_{3}=-2.1\end{array}$ & (c) \\
\hline $\begin{array}{l}o a^{-} \\
\left(B^{\prime}>B\right)\end{array}$ & {$\left[\begin{array}{ccc}0.5 & 2.0 & 0 \\
1.5 & 1.0 & 0 \\
2.0 & -2.0 & -0.5\end{array}\right]$} & $\begin{array}{c}\bar{\lambda}_{2}=1.0, \bar{\lambda}_{3}=-3.0 \\
\lambda_{2}=\lambda_{3}=-1.0\end{array}$ & (d) \\
\hline $\begin{array}{l}\text { III } \\
\left(B^{\prime}=B\right)\end{array}$ & {$\left[\begin{array}{rrr}1.5 & -0.5 & 1.0 \\
-0.5 & 1.5 & -1.0 \\
1.0 & -1.0 & 0.6\end{array}\right]$} & $\begin{array}{l}\bar{\lambda}_{2}=2.96, \bar{\lambda}_{3}=-2.16 \\
\lambda_{2}=2.96, \lambda_{3}=-2.16\end{array}$ & (e) \\
\hline$\left(B^{\prime}>B\right)$ & {$\left[\begin{array}{ccc}1.0 & -0.5 & 0.4 \\
-1.0 & 1.5 & -0.4 \\
-5.0 & 5.0 & 0.5\end{array}\right]$} & $\begin{array}{c}\bar{\lambda}_{2}=6.13, \bar{\lambda}_{3}=-3.13 \\
\lambda_{2}=1.5+2.78 i \\
\quad \text { (complex) } \\
\lambda_{3}=1.5-2.78 i \\
\text { (complex) }\end{array}$ & (f) \\
\hline
\end{tabular}

(e) Model parameters for partially stable INC $\left(C_{i j k l}^{d} \delta_{k l} \neq 0, \lambda_{1}=0\right)$

\begin{tabular}{|c|c|c|}
\hline$C_{i j k l}$ coefficient & $\begin{array}{l}\text { Spectral } \\
\text { properties }\end{array}$ & $\begin{array}{c}\text { Computation } \\
\text { Results in } \\
\text { Figure } 11\end{array}$ \\
\hline \multirow{2}{*}[\begin{array}{ccc}{1.0}&{-0.5}&{0}\\
{-0.5}&{5.0}&{0}\\
{0}&{0}&{1.0}\end{array}]{} & $\lambda_{2}=3.5, \lambda_{3}=2.0$ & \multirow{2}{*}{ (a) } \\
\hline & $\begin{array}{l}\bar{\lambda}_{1}=3.77, \bar{\lambda}_{2}= \\
2.0, \bar{\lambda}_{3}=-0.27\end{array}$ & \\
\hline \multirow{3}{*}[\begin{array}{ccc}{1.0}&{0.5}&{-1.0}\\
{1.0}&{2.0}&{1.0}\\
{1.0}&{-1.0}&{0.75}\end{array}]{} & $\begin{array}{c}\lambda_{2}=1.125+1.96 i \\
\text { (complex) }\end{array}$ & \multirow{3}{*}{ (b) } \\
\hline & $\begin{aligned} \lambda_{3}= & 1.125-1.96 i \\
& (\text { complex })\end{aligned}$ & \\
\hline & $\begin{array}{c}\bar{\lambda}_{1}=1.5, \bar{\lambda}_{2}= \\
0.9, \bar{\lambda}_{3}=-0.155\end{array}$ & \\
\hline
\end{tabular}

Figure 8 and Table $3(\mathrm{~b})$ show four cases of unstable INC, which corresponds to $0 \geq$ $\bar{\lambda}_{2} \geq \overline{\mathcal{\lambda}}_{3}$. In contrast to Figure 7, unstable IC is possible along a few directions. Figures 8(a) and 8 (b) show four and two unstable IC (UIC), respectively, which correspond to $\cos 2 \theta_{\text {inc }}=-1$, and fall within Domain II $\left(0 \geq \bar{\lambda}_{2} \geq \bar{\lambda}_{3}\right.$ and $\lambda_{2} \neq \lambda_{3}<0$ and along boundary $a^{-} A^{-}\left(0 \geq \bar{\lambda}_{2} \geq \bar{\lambda}_{3}\right.$ and $\lambda_{2}=\lambda_{3}$ ) in Figure 6. Figure 8(c) shows the particular case of unstable IC in all directions, which is available only if $B^{\prime}=B$, corresponding to boundary $O A^{-}\left(\lambda_{2}=\lambda_{3}=\bar{\lambda}_{2}=\bar{\lambda}_{3}<\right.$ 0 ) in Figure 6. Finally, Figure 8(d) shows that there is unstable INC in all directions, which corresponds to Domain VII in Figure 6. 


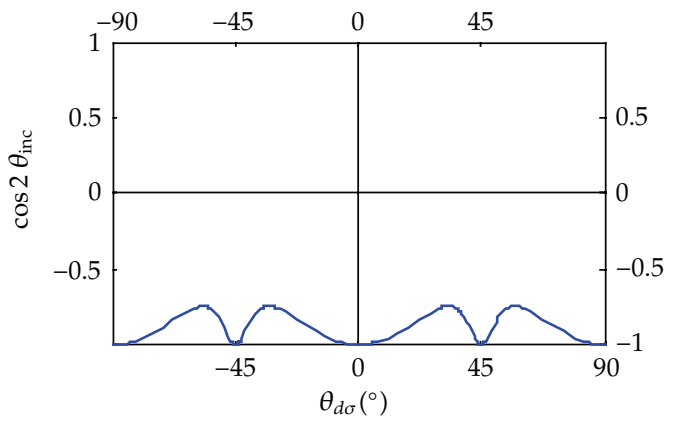

(a) 4 UIC

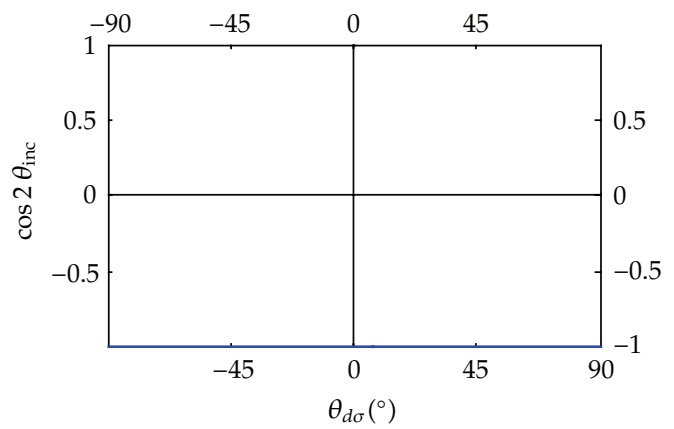

(c) UIC

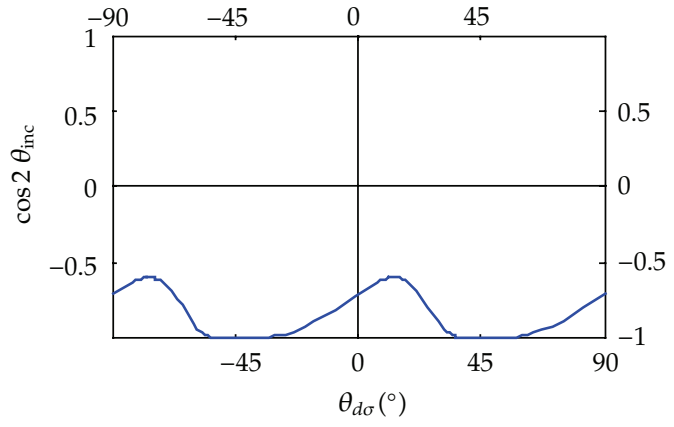

(b) 2 UIC

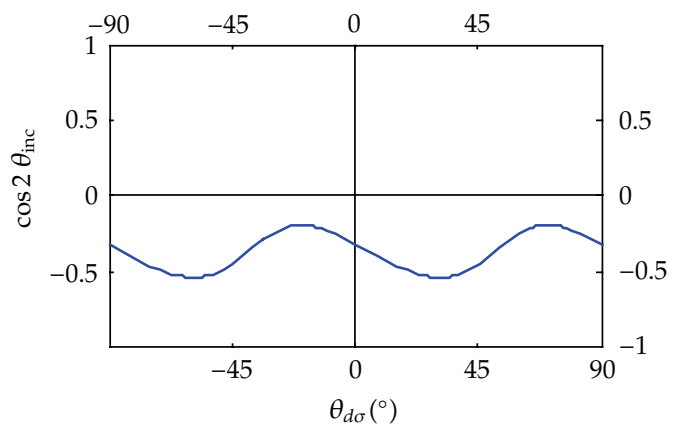

(d) No UIC

Figure 8: $\theta_{d \sigma}-\cos 2 \theta_{\text {inc }}$ for unstable INC $\left(C_{i j k l}^{d} \delta_{k l}=0\right)$.

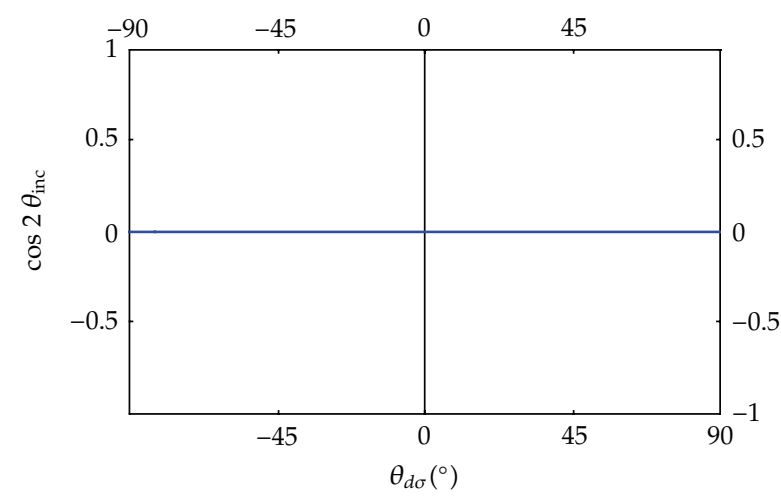

No SIC/UIC

Figure 9: $\theta_{d \sigma}-\cos 2 \theta_{\text {inc }}$ for stable-unstable transition INC $\left(C_{i j k l}^{d} \delta_{k l}=0\right.$, complex $\left.\lambda_{2}, \lambda_{3}\right)$.

Figure 9 and Table $3(\mathrm{c})$ shows a particular case when $\cos 2 \theta_{\text {inc }}=0$, which corresponds to $\bar{\lambda}_{2}=\bar{\lambda}_{3}=0$ and conjugate complex $\lambda_{2}$ and $\lambda_{3}$. Clearly, there is INC in all directions, which corresponds to Point $O^{\prime}$ in Figure 6.

Figure 10 and Table $3(\mathrm{~d})$ show six cases of partially stable INC, which corresponds to $\bar{\lambda}_{2}>0>\bar{\lambda}_{3}$. The case $\bar{\lambda}_{2}>0>\bar{\lambda}_{3}$ can be obtained for any values of $\lambda_{2}$ and $\lambda_{3}$. The classifications correspond to Domains III, IV, V, VIII and boundaries $\mathrm{Oa}^{+}$and $\mathrm{Oa}^{-}$. Stable IC 


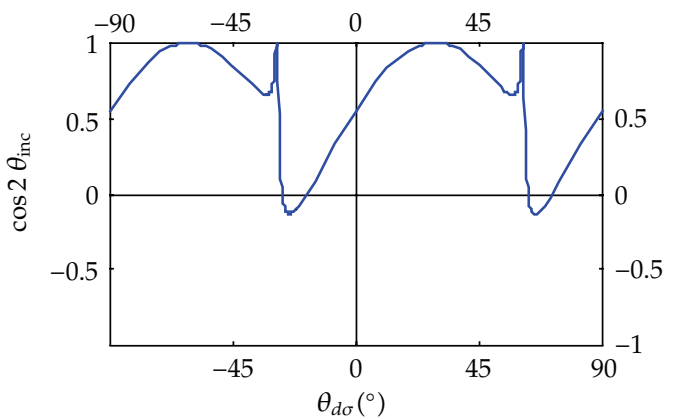

(a) 4 SIC

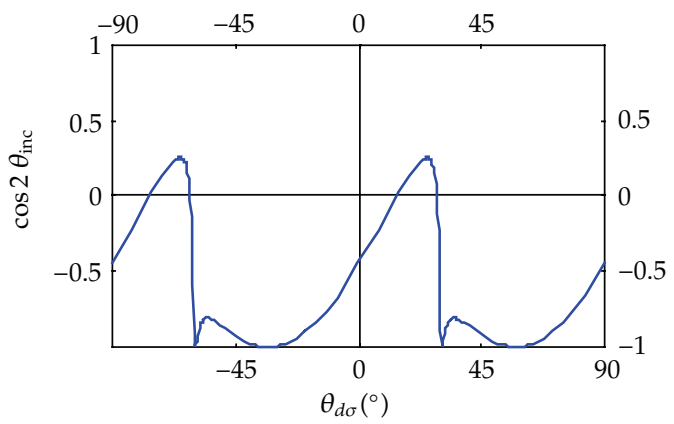

(c) 4 UIC

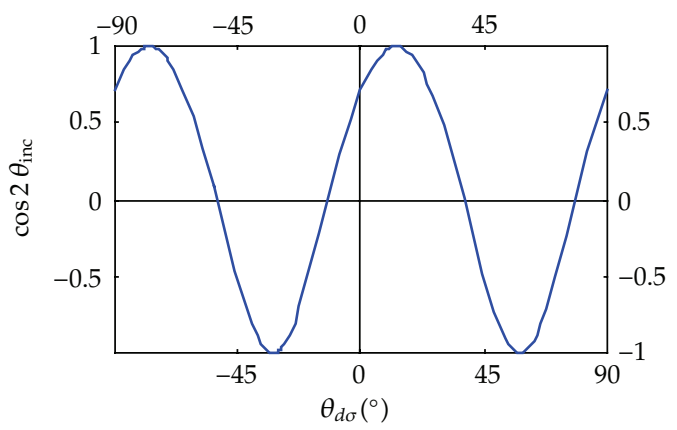

(e) 2 SIC and 2 UIC

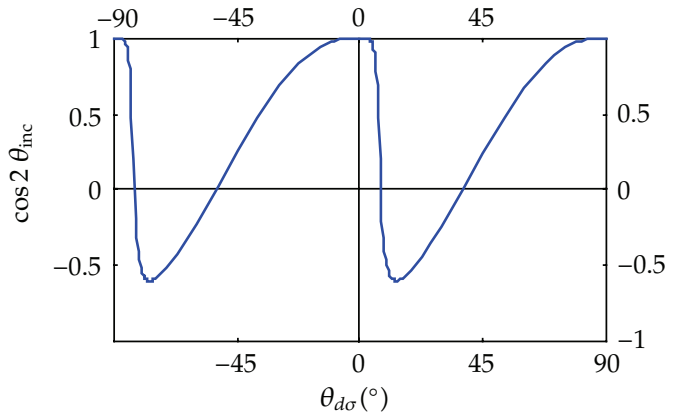

(b) 2 SIC

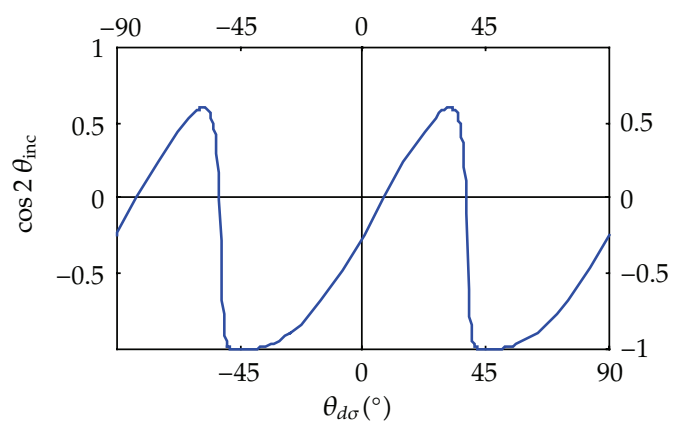

(d) 2 UIC

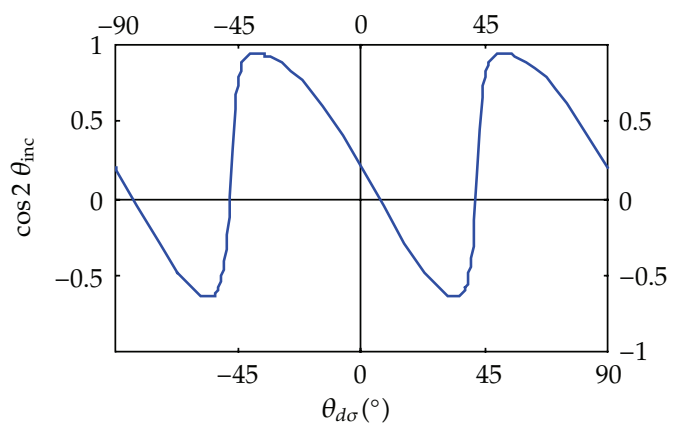

(f) No SIC/UIC

Figure 10: $\theta_{d \sigma}-\cos 2 \theta_{\text {inc }}$ for partially stable INC $\left(C_{i j k l}^{d} \delta_{k l}=0\right)$.

(SIC) can be obtained in 4 directions in Figure 10(a), 2 in Figure 10(b), and 2 in Figure 10(e). Unstable IC (UIC) can be obtained in 4 directions in Figure 10(c), 2 in Figure 10(d) and 2 in Figure 10(e). Figure 10(f) shows that partially stable INC is obtained for all directions, which corresponds to Domain VIII in Figure 6.

As shown in Figures 7-10, INC is independent of $R_{d \sigma}$, which results from $C_{i j k l}^{d} \delta_{k l}=$ 0 . On the other hand, Figure 11 presents the variation of INC in terms $R_{d \sigma}$ and $\theta_{d \sigma}$, when $C_{i j k l}^{d} \delta_{k l} \neq 0$. The intensity of shaded contours indicates the stability of INC. Table 3(e) lists the corresponding material coefficients. In this case, INC cannot be classified using spectral classification, because it depends not only on the principal direction $\theta_{d \sigma}$ but also on the ratio of principal stress increments, $R_{d \sigma}$. Similar results are founds when $\lambda_{2}$ and $\lambda_{3}$ are real and complex. 


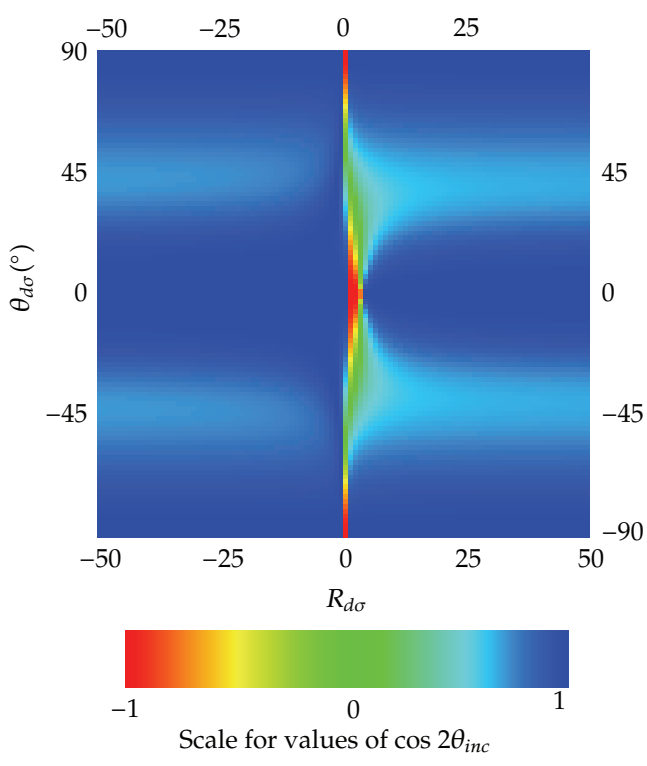

(a) No SIC/UIC (real $\lambda_{2}, \lambda_{3}$ )

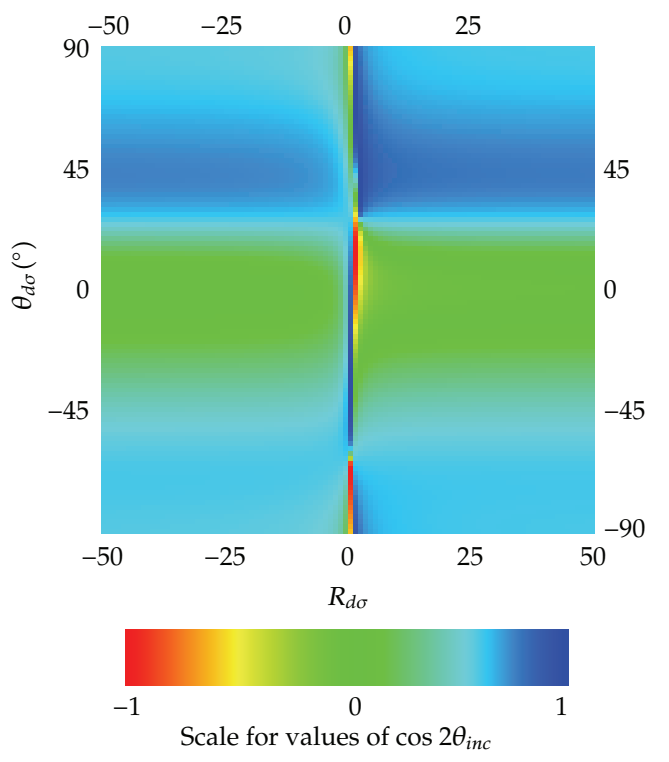

(b) No SIC/UIC (complex $\lambda_{2}, \lambda_{3}$ )

Figure 11: $R_{d \sigma}-\theta_{d \sigma}-\cos 2 \theta_{\text {inc }}$ for partially stable INC $\left(C_{i j k l}^{d} \delta_{k l} \neq 0\right)$.

In the above examples, the compliance matrices $C_{i j k l}$ are clearly related to classical anisotropic materials. For instance, isotropic elasticity always creates stable IC $\left(\lambda_{2}=\lambda_{3}=\right.$ $\left.\bar{\lambda}_{2}=\bar{\lambda}_{3} \geq 0\right)$, while orthogonally anisotropy may create stable INC $\left(\lambda_{2}=\bar{\lambda}_{2} \neq \lambda_{3}=\bar{\lambda}_{3}>0\right.$ and $\left.C_{i j k l}^{d} \delta_{k l}=0\right)$ presented in Figure $7\left(\right.$ a), or partially stable INC $\left(C_{i j k l}^{d} \delta_{k l} \neq 0\right)$ as shown in Figure 11(a). On the other hand, the coefficients of compliance matrix $C_{i j k l}$ for elastoplastic materials may change and generate several distinct eigenvalue cases with the development of work hardening (softening).

In addition, as shown in Figures 7 and 8, pure stable (or unstable) INC that requires the condition of $C_{i j k l}^{d} \delta_{k l}=0$, may be reproduced by Tresca-type or Mises-type models, which are generally used to simulate incompressible material behaviors. However, pressure sensitive materials $\left(C_{i j k l}^{d} \delta_{k l} \neq 0\right)$ create partially stable INC only, as shown in Figure 11.

\section{Conclusion}

The present study has presented a spectral approach to explore the non-coaxial aspects of incremental material behavior and has classified the non-coaxiality between stress and strain increments. It expands the definition of non-coaxiality (NC), which usually refers to the difference between the principal directions of incremental strain and stress state in the solid mechanics literature. The analysis has investigated the incremental non-coaxiality (INC) induced by incremental stress-strain relations, for example, linearized rate-type solids. Based on the concept of deviatoric second-order work, this study has examined the relations between incremental non-coaxiality and stability. It has proposed three classifications that are based on eigenvalues of the constitutive compliance matrix. These classifications distinguish various degrees of incremental non-coaxiality and stability. They determine the conditions for 
which stress and strain increments are collinear and result into stability, instability, and stableinstable transition. The classifications have been illustrated using examples of constitutive matrices that are relevant to elastic as well as elastoplastic constitutive modeling. The present classifications are useful for examining the incremental stability of incompressible materials and determining anisotropic state and optimal material orientation.

\section{Appendix}

The incremental stress-strain relationship given by (2.1) can be rewritten as follows:

$$
\begin{aligned}
& d \varepsilon_{x x}=C_{x x x x} d \sigma_{x x}+C_{x x y y} d \sigma_{y y}+C_{x x x y} d \sigma_{x y} \\
& d \varepsilon_{y y}=C_{y y x x} d \sigma_{x x}+C_{y y y y} d \sigma_{y y}+C_{y y x y} d \sigma_{x y} \\
& d \varepsilon_{x y}=C_{x y x x} d \sigma_{x x}+C_{x y y y} d \sigma_{y y}+C_{x y x y} d \sigma_{x y} .
\end{aligned}
$$

The principal direction of strain increment can be defined as

$$
\tan 2 \theta_{d \varepsilon}=\frac{2 d \varepsilon_{x y}}{d \varepsilon_{x x}-d \varepsilon_{y y}}
$$

Substitution of (A.1), (A.2) and (A.3) into (A.4) leads to

$$
\tan 2 \theta_{d \varepsilon}=\frac{2 C_{x y x x} d \sigma_{x x}+2 C_{x y y y} d \sigma_{y y}+2 C_{x y x y} d \sigma_{x y}}{\left(C_{x x x x}-C_{y y x x}\right) d \sigma_{x x}+\left(C_{x x y y}-C_{y y y y}\right) d \sigma_{y y}+\left(C_{x x x y}-C_{y y x y}\right) d \sigma_{x y}} .
$$

Invoking that stress is related to its principal values by

$$
\begin{aligned}
& d \sigma_{x x}=\frac{d \sigma_{1}+d \sigma_{2}}{2}+\frac{d \sigma_{1}-d \sigma_{2}}{2} \cos 2 \theta_{d \sigma} \\
& d \sigma_{y y}=\frac{d \sigma_{1}+d \sigma_{2}}{2}-\frac{d \sigma_{1}-d \sigma_{2}}{2} \cos 2 \theta_{d \sigma} \\
& d \sigma_{x y}=\frac{d \sigma_{1}-d \sigma_{2}}{2} \sin 2 \theta_{d \sigma} .
\end{aligned}
$$

With the introduction of (A6), (A.7) and (A.8) into (A.5), thus (A.5) can be restated as

$$
\tan 2 q_{d e}=\frac{2 C_{x y x x} \mathcal{A}+2 C_{x y y y} C+2 C_{x y x y} \boldsymbol{\Phi}}{\left(C_{x x x x}-C_{y y x x}\right) \mathcal{A}+\left(C_{x x y y}-C_{y y y y}\right) \mathcal{C}+\left(C_{x x x y}-C_{y y x y}\right) \boldsymbol{\Phi}}
$$

where $\mathcal{A}, \mathcal{C}$, and $\boldsymbol{\Phi}$ denote $\left(R_{d \sigma}+\tan ^{2} \theta_{d \sigma}\right),\left(R_{d \sigma} \tan ^{2} \theta_{d \sigma}+1\right)$, and $\left(R_{d \sigma} \tan \theta_{d \sigma}-\tan \theta_{d \sigma}\right)$, respectively. Recall that $R_{d \sigma}=d \sigma_{1} / d \sigma_{2}$ in (A.9). Based on (A.9), we can concluded (2.4).

Analogous to the above analysis, it can be checked that it is also true for (2.5). 


\section{Acknowledgment}

The financial support provided by National Natural Science Foundation of China (Grant nos. 10972159 and 50825803) and Natural Science Foundation of Shanghai, China (Grant no. 08ZR1420100).

\section{References}

[1] J. W. Rudnicki and J. R. Rice, "Conditions for the localization of deformation in pressure-sensitive dilatant materials," Journal of the Mechanics and Physics of Solids, vol. 23, no. 6, pp. 371-394, 1975.

[2] E. Papamichos and I. Vardoulakis, "Shear band formation in sand according to non-coaxial plasticity model," Géotechnique, vol. 45, no. 4, pp. 649-661, 1995.

[3] J. G. Qian, J. Yang, and M. S. Huang, "Three-dimensional noncoaxial plasticity modeling of shear band formation in geomaterials," Journal of Engineering Mechanics, vol. 134, no. 4, pp. 322-329, 2008.

[4] K. H. Roscoe, "The influence of strain in soil mechanics," Géotechnique, vol. 20, pp. 129-170, 1970.

[5] M. Gutierrez, K. Ishihara, and I. Towhata, "Flow theory for sand during rotation of principal stress direction," Soils and Foundations, vol. 31, no. 4, pp. 121-132, 1991.

[6] A. Drescher and G. de Josselin de Jong, "Photoelastic verification of a mechanical model for the flow of a granular material," Journal of the Mechanics and Physics of Solids, vol. 20, no. 5, pp. 337-340, 1972.

[7] M. Oda, J. Konishi, and S. Nemat-Nasser, "Experimental micromechanical evaluation of strength of granular materials: effects of particle rolling," Mechanics of Materials, vol. 1, no. 4, pp. 269-283, 1982.

[8] R. Hill, The Mathematical Theory of Plasticity, The Clarendon Press, Oxford, UK, 1950.

[9] I. Vardoulakis and B. Graf, "Calibration of constitutive models for granular materials using data from biaxial experiments," Géotechnique, vol. 35, pp. 299-317, 1985.

[10] Z. Mroz, "An attempt to describe the behavior of metals under cyclic loads using a more general work hardening model," Acta Mecanica, vol. 7, pp. 199-212, 1969.

[11] W. Wu, "Rational approach to anisotropy of sand," International Journal for Numerical and Analytical Methods in Geomechanics, vol. 22, no. 11, pp. 921-940, 1998.

[12] X. S. Li and Y. F. Dafalias, "A constitutive, framework for anisotropic sand including non-proportional loading," Géotechnique, vol. 54, no. 1, pp. 41-55, 2004.

[13] A. J. M. Spencer, "A theory of the kinematics of ideal soils under plane strain conditions," Journal of the Mechanics and Physics of Solids, vol. 12, pp. 337-351, 1964.

[14] G. de Josselin de Jong, "The double sliding, free rotating model for granular assemblies," Géotechnique, vol. 21, pp. 155-162, 1971.

[15] J. A. M. Teunissen, "On double shearing in frictional materials," International Journal for Numerical and Analytical Methods in Geomechanics, vol. 31, no. 1, pp. 23-51, 2007.

[16] M. Oda, "Inherent and induced anisotropy in plasticity theory of granular soils," Mechanics of Materials, vol. 16, no. 1-2, pp. 35-45, 1993.

[17] S. Nemat-Nasser, "A micromechanically-based constitutive model for frictional deformation of granular materials," Journal of the Mechanics and Physics of Solids, vol. 48, no. 6-7, pp. 1541-1563, 2000.

[18] H. S. Yu, "Non-coaxial theories of plasticity for granular materials," in Proceedings of the 12th International Association for Computer Methods and Advances in Geomechanics (IACMAG '08), pp. 361378, Goa, India, 2008.

[19] R. K. S. Wong and J. R. F. Arthur, "Induced and inherent anisotropy in sand," Géotechnique, vol. 35, no. 4, pp. 471-481, 1985.

[20] K. Miura, S. Miura, and S. Toki, "Deformation behavior of anisotropic dense sand under principal stress axes rotation," Soils and Foundations, vol. 26, no. 1, pp. 36-52, 1986.

[21] H. S. Yu and X. Yuan, "On a class of non-coaxial plasticity models for granular soils," Proceedings of the Royal Society A, vol. 462, no. 2067, pp. 725-748, 2006.

[22] S. Tsutsumi and K. Hashiguchi, "General non-proportional loading behavior of soils," International Journal of Plasticity, vol. 21, no. 10, pp. 1941-1969, 2005.

[23] G. Mandl and R. F. Luque, "Fully developed plastic flow of granular materials," Géotechnique, vol. 20, pp. 277-307, 1970.

[24] A. N. Norris, "Optimal orientation of anisotropic solids," The Quarterly Journal of Mechanics and Applied Mathematics, vol. 59, no. 1, pp. 29-53, 2006. 


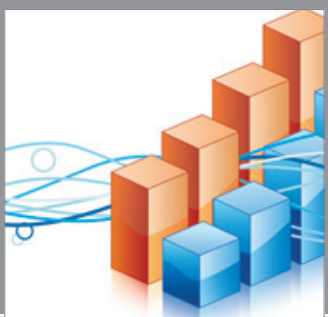

Advances in

Operations Research

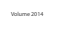

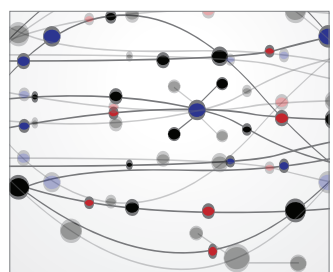

\section{The Scientific} World Journal
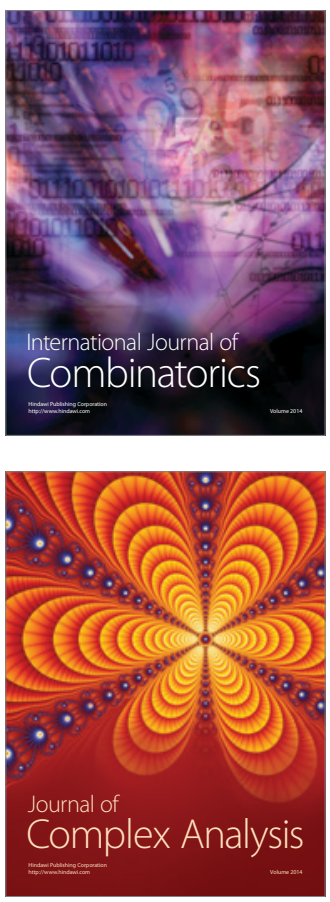

International Journal of

Mathematics and

Mathematical

Sciences
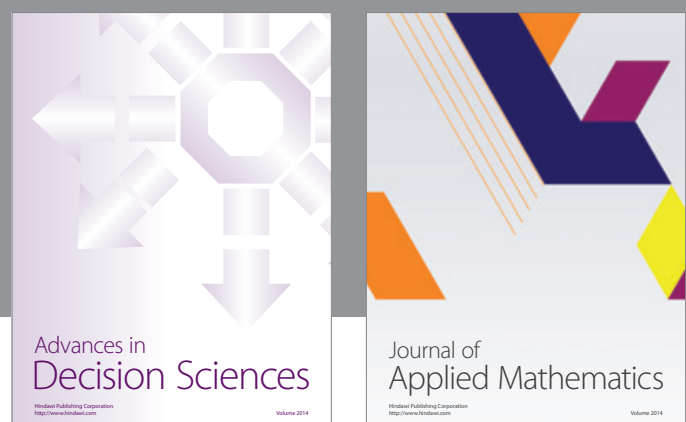

Journal of

Applied Mathematics
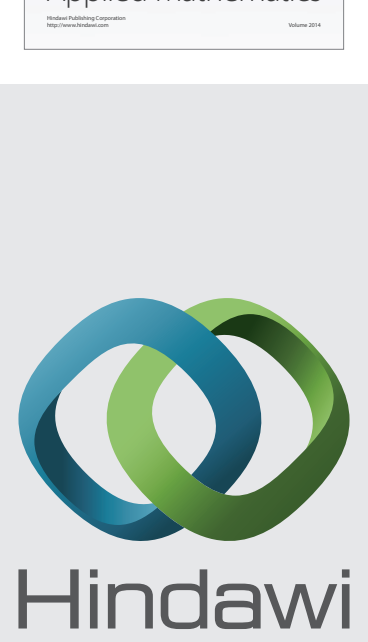

Submit your manuscripts at http://www.hindawi.com
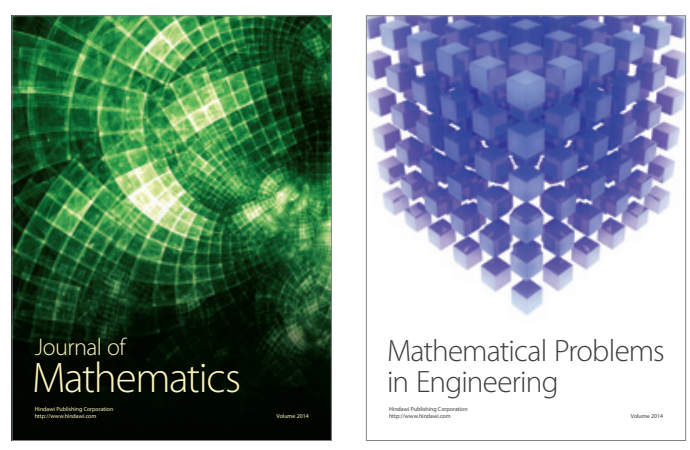

Mathematical Problems in Engineering
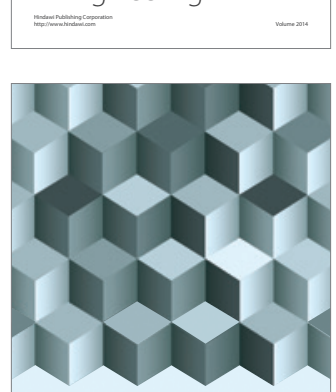

Journal of

Function Spaces
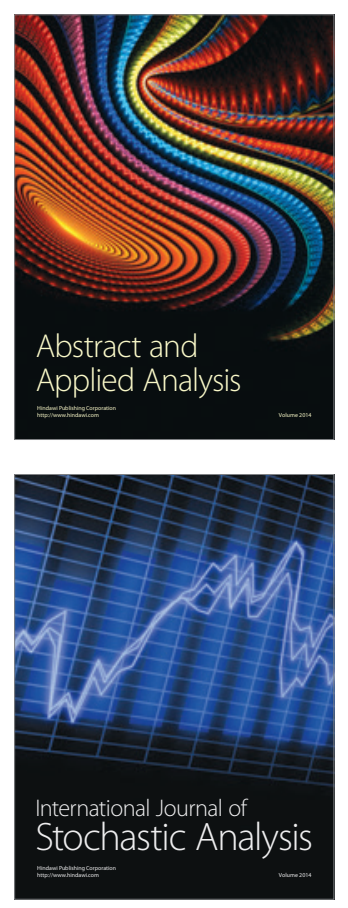

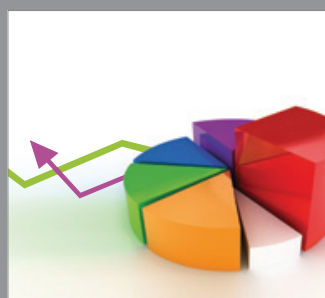

ournal of

Probability and Statistics

Promensencen
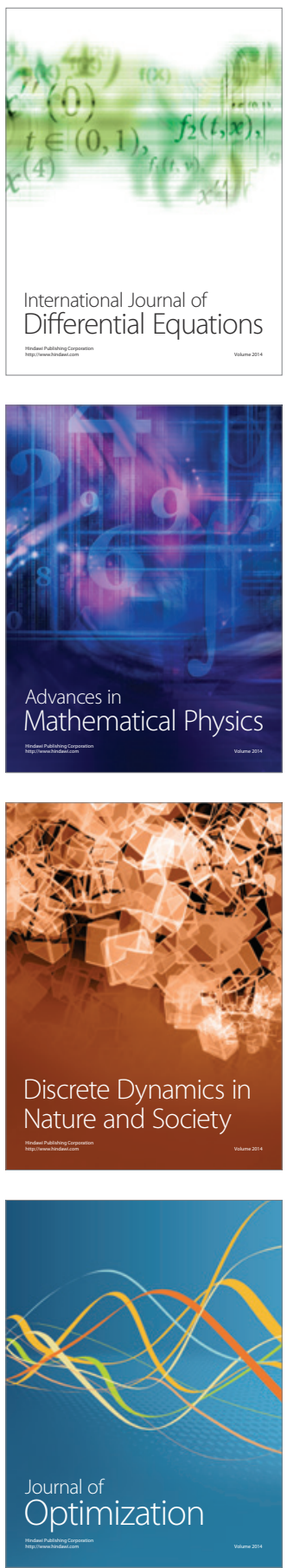